\title{
A Data-Driven Multi-field Analysis of Nanocomposites for Hydrogen Storage
}

\author{
John Michopoulos, Nick Tran, and Sam Lambrakos \\ Materials Science and Component Technology Directorate, \\ U.S. Naval Research Laboratory, \\ Washington DC, 20375,U.S.A \\ \{john.michopoulos, nick.tran, sam.lambrakos\}@nrl.navy.mil
}

\begin{abstract}
This paper focuses on computational parameter identification associated with heat and mass diffusion macro-behavioral models of hydrogen storage systems from a continuum multiphysics perspective. A single wall nanotube (SWNT) based composite pellet is considered as our representative finite continuum system. The corresponding partial differential equations (PDEs) governing the spatio-temporal distribution of temperature and hydrogen concentration are formulated. Analytical solutions of the system of coupled PDEs are constructed and utilized in the context of inverse analysis. The corresponding non-linear optimization problem is formulated in order to determine the unknown parameters of the model, based on an objective function and constraints consistent with experimentally acquired data along with the physical and utilization requirements of the problem. Behavioral simulation results are presented in an effort to demonstrate the applicability of the methodology. Finally, we indicate potential extensions of this methodology to multi-scale and manufacturing process optimization.
\end{abstract}

\section{Introduction}

The activities described herein are a part of a larger effort associated with the development of a data-driven environment for multiphysics applications (DDEMA) $[1,2,3,4]$. The analytical methodology for approaching system identification problems is based on establishing a corresponding inverse problem that can be solved by means of global optimization as shown earlier $[5,6,7]$ for various problems ranging from material nonlinear constitutive response to welding characterizations.

The recent utilization of SWNTs as an essential component for nanocomposites for hydrogen storage has lead to the direct need for modeling the behavioral characteristics of such systems during both the hydriding and dehydriding stages. The desire for manufacturing highly efficient, inexpensive and long lasting hydrogen storage systems underscores our motivation for modeling such system as a special case of our general effort.

In order to achieve these goals, we proceeded with the modeling of a nanocomposite pellet's hydriding and dehydriding response coupled with heat conduction 
and chemical reactivity under the framework of an inverse problem setting applied to preliminary experiments that generated the data to drive our modeling. Continuum multi-field modeling efforts have been based on continuum thermodynamics and conservation theorems $[8,9,10]$. Here we present the results of our multi-field modeling effort [5] for the problem at hand along with a description of the associated solutions.

\section{Behavioral Modeling of Hydrogen Storage Pellet}

Consider a medium with isotropic and homogeneous aggregate properties at the macro-length scale. Furthermore, consider that this medium is exposed to temperature and multi-species concentration boundary conditions. As heat diffusion proceeds in the medium so does multi-species mass diffusion. The species may or may not be chemically reactive with the host medium or with each other. A medium under such a multi-field excitation includes as a special case the SWNT-enhanced materials considered for hydrogen storage. The general procedure for deriving the continuum multiphysics model for this system is analogous to the one followed for hygrothermoelastic composites elsewhere [10]. The resulting general system of PDEs [11] describes all open continuum systems under the influence of temperature, and multi-species diffusion with chemical reactions among the species. However, for the case of the hydrogen storage continua of the type used here we do not have cross-species reactivity since hydrogen is the only species involved. The diffused hydrogen only reacts with the matrix and the embedded SWNTs of the composite. This process can be abstracted as an absorption/desorption diffusion. Therefore, as a first approximation we will assume a single component system (i.e. we will consider the concentration of hydrogen $C$ as our mass concentration variable) with no chemical reaction involved and $T$ as our temperature field state variable. In this case the following pair of coupled PDEs is valid:

$$
\begin{aligned}
& \frac{\partial C}{\partial t}=D_{m} \nabla^{2} C+\lambda D_{h} \nabla^{2} T, \\
& \frac{\partial T}{\partial t}=\nu D_{m} \nabla^{2} C+D_{h} \nabla^{2} T .
\end{aligned}
$$

Here, $D_{m}$ and $D_{h}$ are the mass and heat diffusivities, respectively, and $\lambda, \nu$ are both coupling coefficients. This heat-mass diffusion system is completed by the generalized boundary conditions (of the third kind),

$$
\begin{array}{r}
T(\mathbf{x}, t)+\frac{k_{h}(\mathbf{x})}{h_{T}} \frac{\partial T(\mathbf{x}, t)}{\partial \mathbf{n}}=\phi_{T}(\mathbf{x}, t), \mathbf{x} \in S \\
C(\mathbf{x}, t)+\frac{k_{m}(\mathbf{x})}{h_{C}} \frac{\partial C(\mathbf{x}, t)}{\partial \mathbf{n}}=\phi_{C}(\mathbf{x}, t), \mathbf{x} \in S
\end{array}
$$

where $S$ represents the boundary, $\mathbf{x}$ and $\mathbf{n}$ the position and outward normal vectors on the boundary, respectively, $k_{h}, k_{m}$ are the heat and mass conductivities, respectively, $h_{T}, h_{C}$ are the heat and mass transfer coefficients respectively, 
and finally $\phi_{T}(\mathbf{x}, t), \phi_{C}(\mathbf{x}, t)$ are the prescribed distributions of temperature and mass concentration on the boundary, respectively. Boundary conditions of the first and second kinds can be produced by appropriate omission of the second terms (first kind) and the first terms (second kind) of these equations. The cylindrical symmetry of the SWNT composite pellet suggests transforming the governing equations from the cartesian coordinate system to a cylindrical frame of reference $(\{x, y, z, t\} \rightarrow\{r, \vartheta, z, t\})$. In addition, the axisymmetric character of the applied boundary conditions further simplify the system of Eqs. (1) by coordinate transformation of the nabla operators. It has been shown elsewhere [10] that it is possible to uncouple the system of Eqs. (1) by using the solutions of the uncoupled PDEs if $\lambda, \nu$ are constants via the method of normal coordinates. One form of the uncoupled solution is given by

$$
\begin{array}{r}
T=T_{0}+\left(T_{f}-T_{0}\right) F_{1}\left(r / r_{o}, z / l_{o}, D_{h} t / r_{o}^{2}, D_{h} t / l_{o}^{2}, u_{d}\right) \\
+\nu u_{d}\left(C_{f}-C_{0}\right) F_{2}\left(r / r_{o}, z / l_{o}, D_{m} t / r_{o}^{2}, D_{m} t / l_{o}^{2}, 1 / u_{d}\right) \\
C=C_{0}+\left(C_{f}-C_{0}\right) F_{1}\left(r / r_{o}, z / l_{o}, D_{m} t / r_{o}^{2}, D_{m} t / l_{o}^{2}, 1 / u_{d}\right) \\
+\lambda\left(T_{f}-T_{0}\right) F_{2}\left(r / r_{o}, z / l_{o}, D_{h} t / r_{o}^{2}, D_{h} t / l_{o}^{2}, u_{d}\right)
\end{array}
$$

where $T_{f}, C_{f}$ are the final values of the temperature and hydrogen concentrations, respectively, that are applied on the boundary and reached by the continuum at equilibrium conditions and where,

$$
F_{i}\left(r, z, \tau_{r}, \tau_{z}, u\right)=\Psi_{i}^{c}\left(r, \tau_{r}, u\right) \Psi_{i}^{s}\left(z, \tau_{z}, u\right) .
$$

The solutions $F_{i}$ for the finite cylinder geometry have been constructed here as the product of the two one dimensional solutions corresponding to an infinite cylinder of radius $r_{o}$ and an infinite slab of thickness $2 l_{o}$ with appropriate scaling of time via usage of $\tau_{r}$ and $\tau_{z}$, respectively. The functions $\Psi_{i}^{j}$ with $j=c, s$ and $i=1,2$ are defined by

$$
\begin{gathered}
\Psi_{1}^{j}\left(x_{j}, \tau_{j}, u\right)=\left[1-H_{1}^{j}(u)\right] \Psi^{j}\left(x_{j}, D_{2}(u) \tau_{j}\right)+H_{1}^{j}(u) \Psi^{j}\left(x_{j}, D_{1}(u) \tau_{j}\right) \\
\Psi_{2}^{j}\left(x_{j}, \tau_{j}, u\right)=H_{2}^{j}(u)\left[\Psi^{j}\left(x_{j}, D_{2}(u) \tau_{j}\right)-\Psi^{j}\left(x_{j}, D_{1}(u) \tau_{j}\right)\right],
\end{gathered}
$$

where we have used the contractions,

$$
\begin{gathered}
H_{1}\left(u_{d}\right)=\frac{1}{2}\left[1-\left(1-u_{d}\right) H_{2}\left(u_{d}\right)\right. \\
H_{2}\left(u_{d}\right)=\frac{D_{1} D_{2}}{\left(D_{2}-D_{1}\right) u_{d}},
\end{gathered}
$$

where $u_{d}=D_{m} / D_{h}$ and

$$
\begin{aligned}
& D_{1}=2 u_{d} /\left[1+u_{d}+\sqrt{\left(1-u_{d}\right)^{2}-4 u_{d}(1-\lambda \nu)}\right] \\
& D_{2}=2 u_{d} /\left[1+u_{d}-\sqrt{\left(1-u_{d}\right)^{2}-4 u_{d}(1-\lambda \nu)}\right] .
\end{aligned}
$$


Functions $\Psi^{j}$ are the solutions of the normalized one-dimensional problems for the cylinder $m=0$ and the slab $m=1 / 2$ and are defined by

$$
\Psi^{j}(x, \tau)=1-2 \sum_{i=1}^{\infty} \frac{1}{\mu_{i}}\left[1+\frac{2 m}{B i}+\left(\frac{\mu_{i}}{B i}\right)^{2}\right]^{-1} x^{m} \frac{J_{-m}\left(\mu_{i} x\right)}{J_{1-m}\left(\mu_{i}\right)} e^{-\mu_{i}^{2} \tau},
$$

in terms of the Bessel functions $J_{i}$. The coefficients $\mu_{i}$ are the roots of the transcendental equation

$$
B i J_{-m}(\mu)-\mu J_{1-m}=0 .
$$

\section{Inverse Problem Setup}

The set of diffusivities $\left(D_{h}, D_{m}\right)$, the coupling constants $(\lambda, \nu)$ and the four instances of the Biot number $B i\left((B i)_{T}^{c}=h_{T} r_{o} / k_{h},(B i)_{C}^{c}=h_{C} r_{o} / k_{m},(B i)_{T}^{s}=\right.$ $\left.h_{T} l_{o} / k_{h},(B i)_{C}^{s}=h_{C} l_{o} / k_{m}\right)$ determine the behavioral model completely when the geometry and initial and boundary conditions are known. Determination of these constants from experimental data constitutes the inverse problem formulation.

\subsection{Experimental Setup and Associated Data (NT)}

The pellet samples for the hydridingdehydriding study were made by uniaxially cold pressing the Mgx wt.\% Mm powder. The details of manufacturing and processing the pellets are given elsewhere [12,13]. Figure (1) shows the pellet and the experimental setup for hydriding and dehydriding the pellet. Hydriding and dehydriding of Mgx wt.\% Mm pellets were carried out inside a dual reactor made of seamless inconel tubes. Two thermocouples were attached to the outside of each tube and were calibrated to reflect the temperature of the reaction zone. All data from the hydriding and dehydriding process were digitally captured by

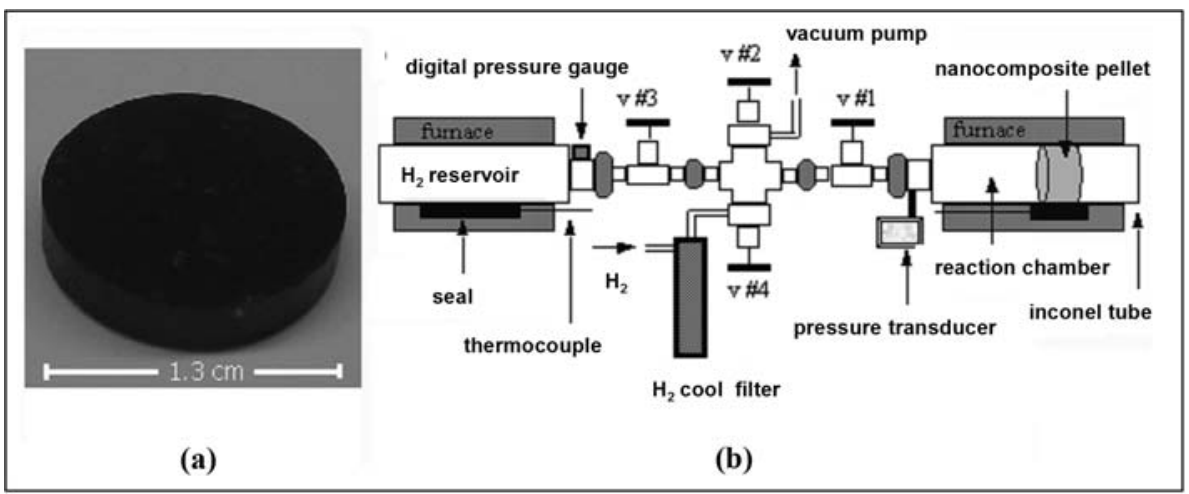

Fig. 1. Hydrorgen storage pellet (a) and experimental setup schematic for hydriding and dehydriding of the pellet (b) 
a data acquisition board, which was controlled by our custom code [13]. The hydrogen uptake or release of the Mgx wt.\% Mm pellets was determined by measuring the pressure changes in the reaction chamber before and after each hydriding or dehydriding treatment.

\subsection{Nonlinear Optimization}

The general outline of using design optimization for parameter identification is a well established discipline. It usually involves the usage of an optimization module that utilizes behavioral data from the actual physical system (collected experimentally) as well as simulated data from a potential model. The design variables to be identified are the constants of the model. Ultimately, the simulated behavior has to reproduce the experimentally observed behavior and therefore an objective function must be minimized. For an objective function $f=f(\mathbf{X})$ with $\mathbf{X}$ being the resultant vector defined by the design variable component vectors such as $x_{1} \mathbf{i}_{1}, \ldots, x_{n} \mathbf{i}_{n} \in \mathbf{X}^{n}$, where $\mathbf{X}^{n}$ is the vector space spanned by the basis vectors $\mathbf{i}_{1}, \ldots, \mathbf{i}_{n}$. For this general case the optimization problem can be expressed by

$$
\begin{gathered}
\min \left[f\left(x_{1}, \ldots, x_{n}\right)\right]_{x_{i} \in \Re^{n}} \\
c_{j}^{e q}\left(x_{1}, \ldots, x_{n}\right)=0, c_{i}^{\text {ineq }}\left(x_{1}, \ldots, x_{n}\right) \geq 0, j=1, \ldots, p
\end{gathered}
$$

Relations (10b) express the equality and inequality constraints.

In our analysis the behavior of the system is described by the time evolutions of the total temperature and hydrogen absorption. The stimulation of the system is expressed by the multi-field boundary conditions that define the temperature and hydrogen mass concentration on the boundary. We define $T_{i}^{\text {sim }}\left(t_{i}\right), C_{i}^{\text {sim }}\left(t_{i}\right)$ and $T_{i}^{\exp }\left(t_{i}\right), C_{i}^{\exp }\left(t_{i}\right)$ to be the simulated and experimental values, respectively, of the temperature and hydrogen concentration at a discrete evaluation point $i=1 \ldots, p$ where $p$ is the total number of evaluations. We can now form the objective function as the sum of the squares of their differences in the least square sense, as follows:

$$
\begin{aligned}
f\left(x_{1}, \ldots, x_{n}\right)= & \sum_{i=1}^{p}\left[T_{i}^{e x p}\left(t_{i}\right)-T_{i}^{s i m}\left(x_{1}, \ldots, x_{n}, t_{i}\right)\right]^{2}+ \\
& +\sum_{i=1}^{p}\left[C_{i}^{\exp }\left(t_{i}\right)-C_{i}^{s i m}\left(x_{1}, \ldots, x_{n}, t_{i}\right)\right]^{2}
\end{aligned}
$$

We set the simulated values to be the average values of the solutions of the PDEs along the edge of the pellet for the temperature and over the entire volume for the hydrogen concentration according to

$$
\begin{gathered}
T_{i}^{s i m}\left(x_{1}, \ldots, x_{n}, t_{i}\right)=\int_{-l_{0}}^{l_{0}} T\left(r_{0}, z, t_{i} ; x_{1}, \ldots, x_{8}\right) d z \\
C_{i}^{s i m}\left(x_{1}, \ldots, x_{n}, t_{i}\right)=2 \pi \int_{0}^{r_{0}} \int_{-l_{0}}^{l_{0}} C\left(r, z, t_{i} ; x_{1}, \ldots, x_{8}\right) d r d z .
\end{gathered}
$$


The integrands in these relations are the ones defined in Eqs. (5) with the special one-to-one substitution $\left\{x_{1}, \ldots, x_{8}\right\} \rightarrow\left\{D_{h}, D_{m}, \lambda, \nu,(B i)_{T}^{c},(B i)_{T}^{s},(B i)_{C}^{c}\right.$, $\left.(B i)_{C}^{s}\right\}$.

\section{$4 \quad$ Preliminary Results}

The implementation of the optimization procedure was formulated in Mathematica [14] with various global nonlinear optimization algorithms encoded in the package Global Optimization "GO-5.0" [15]. The function "GlobalSearch" that yielded the quickest results utilizes a generalized hill climbing technique that is based on Newton's method but uses a generalized gradient rather than a derivative, and allows for analytic linear or nonlinear constraints. Multiple starts are used to test for the existence of multiple solutions. The multiple starts are generated randomly from the region defined by the range of parameter values input by the user. Feasible starting regions are not needed, but it is assumed that objective function values in this region are Real.

The initial and boundary conditions for the computation of the solutions of the PDEs governing the system as required by the simulated values within the objective function, were chosen to correspond to the experimental procedure. Specifically they were $T_{0}=27^{\circ} C, T_{f}=200^{\circ} C, C_{0}=0$ and $C_{f}=1.1$.

Dehydriding data were also considered along with the hydriding data but have not effected the identified parameters.

Figure (2) shows the hydriding time evolution observed experimentally along with the one computed after parameter identification took place. The identified model fits the hydriding experimental data very well and similar performance is observed (but not included here) for the dehydriding data. Therefore, the model may be adopted as a "calibrated" model for simulation of the behavior of similar systems in many other situations and applications, where the shape of specimen varies but the nanocomposite material remains the same.

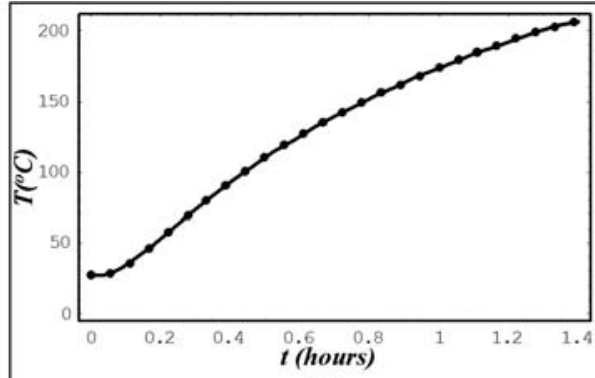

(a)

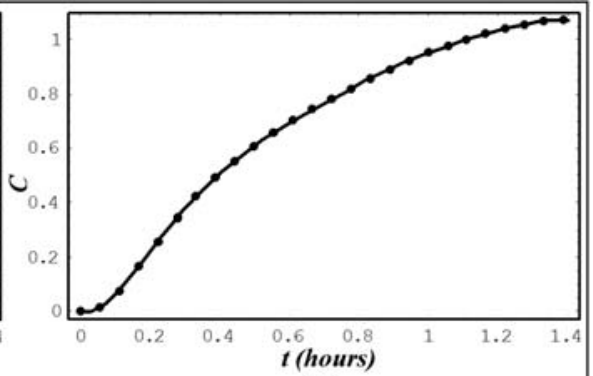

(b)

Fig. 2. Experimental (dots) and simulated (continuous line) after the parameter identification has been conducted, for the time evolution of the temperature (a) and Hydrogen concentration (b) time evolutions during the hydriding process 


\section{$5 \quad$ Potential Extensions}

Careful consideration of the identified parameters implies two distinct correlations. The subset $\left\{D_{h}, D_{m}, \lambda, \nu\right\}$ is related to the characteristic behavior of the nanocomposite system and the macroscopic length scale, while the subset $\left\{(B i)_{T}^{c},(B i)_{T}^{s},(B i)_{C}^{c},(B i)_{C}^{s}\right\}$ is related to the characteristic behavior of the reactor-nanocomposite system owing to the fact that Biot numbers are functions of the heat and mass transfer coefficients that in turn depend on the fluid properties of the gas (hydrogen) according to the Dittus-Boelter equation. These are controllable by the reactor/furnace setup. This suggests that the above referenced optimization procedure is capable of identifying all parameters of interest to the technology practitioner. Therefore, a natural extension of this technique could include any parameters that participate in a given utility (efficiency of performance), aging, or economic model of the system. This extension is well posed as long as the parameters of these models are related to any of the behavioral parameters of the system and an appropriate objective function can be constructed.

In addition to optimizing the usability aspects of such a system one can also optimize the condition of manufacturing related to desirable performance (from efficiency, economic, aging, etc perspectives) by establishing the process control models associated with the manufacturing of such systems, with the lower length scale behavioral models that may be derived from ab initio modeling methodologies among many others.

\section{Conclusions}

We have utilized general coupled mass and heat diffusion, to construct a PDE model to capture the hydrogen storage behavior of a nanocomposite pellet system. We have formulated the inverse problem approach for utilizing data to create a procedure for the data-driven identification of the free parameters participating in the behavior model. We implemented the computational equivalent of the procedure and performed the identification based on experimental data collected for the hydriding cycle of the nanocomposite pellet system. Comparison of the identified system to the experimental data, demonstrated how well the identification has performed. Finally, two specific motivating extensions were discussed to define the context of our future intensions.

\section{Acknowledgements}

The authors acknowledge the support by the National Science Foundation under grant ITR-0205663. In addition, the authors would like to extend their special thanks to Dr. F. Darema for her constant availability and encouragement throughout this effort. 


\section{References}

1. Michopoulos, J., Tsompanopoulou, P., Houstis, E., Rice, J., Farhat, C., Lesoinne, M., Lechenault, F., DDEMA: A Data Driven Environment for Multiphysics Applications,in: Proceedings of International Conference of Computational Science - ICCS'03, Sloot, P.M.A., et al. (Eds.), LNCS 2660, Part IV, Springer-Verlag, Haidelberg, (2003) 309-318.

2. Michopoulos, J., Tsompanopoulou, P., Houstis, E., Rice, J., Farhat, C., Lesoinne, M., Lechenault, F., Design Architecture of a Data Driven Environment for Multiphysics Applications, in: Proceedings of DETC'03, Paper No DETC2003/CIE48268, (2003).

3. Michopoulos, J., Tsompanopoulou, P., Houstis, E., Farhat, C., Lesoinne, M., Rice, J., Joshi, A., On a Data Driven Environment for Multiphysics Applications, Future Generation Computer Systems, in-print (2005).

4. Michopoulos,J., Tsompanopoulou, P., Houstis, E., Joshi A., Agent-based Simulation of Data-Driven Fire Propagation Dynamics, in: Proceedings of International Conference of Computational Science - ICCS'04, Bubak, M., et al. (Eds.), LNCS 3038, Part III, Springer-Verlag, Haidelberg, (2004) 732-739.

5. Michopoulos, J., Computational Modeling of Multi-Field Ionic Continuum Systems, in: Proceedings of International Conference of Computational Science ICCS'04, Bubak, M., et al. (Eds., LNCS 3039, Part IV, Springer-Verlag, Haidelberg, (2004) 621-628.

6. Michopoulos, J., Automated Characterization of Material Constitutive Response, in: Proceedings of Sixth World Congress of Computational Mechanics (WCCM-VI 2004), Tsinghua University Press \& Springer-Verlag, Haidelberg, (2004) 486-491.

7. Lambrakos, S.G., Milewski, J.O., Analysis of Processes Involving Heat Deposition Using Constrained Optimization, Sci. and Tech. of Weldong and Joining, 7(3), (2002), 137.

8. Truesdell, C., Toupin, R., 1960, "The Classical Field Theories", in Handbuch der Physik ( Herausgegeben con S. Flugge) III/1, Springer-Verlag, Berlin.

9. Green, A. E., Naghdi, P. M., 1995, "A unified procedure for construction of theories of deformable media. I. Classical continuum physics", Proc. Roy. Soc. London Ser. A 448 (1934), pp. 335-356.

10. Sih, G.C., Michopoulos, J.G., Chou S.C., "Hygrothermoelasticity", Martinus Nijhoff Publishers (now Kluwer Academic), Dordrecht, (1986).

11. Michopoulos, J.,Lambrakos, S.G., Tran, N. E., Multi-Field Characterization of Single Wall Nano-Tube Composites for Hydrogen Storage, ASME-CIE conference, Long Beach, CA, September 24-28, 2005, To appear, (2005).

12. Tran, N. E. , Imam, M. A., Feng, C. R., Evaluation of Hydrogen Storage Characteristics of Magnesium-misch Metal Alloys. J Alloys Comp ,359, (2003), 225-229.

13. Tran, N. E., Lambrakos, S.G., Purification and Defects Elimination of SingleWalled Carbon Nanotubes by Thermal Reduction Technique. To appear.

14. Wolfram, S., The Mathematica Book, Wolfram Media, (2004).

15. Loehle Enterprises, Global Optimization 5.0 for Mathematica, Loehle Enterprises, (2004). 